\title{
Contextualizing motivations and perceived barriers of healthy nutrition and lifestyle behaviours among Malaysian adults with metabolic syndrome
}

MUHAMMAD DANIEL AZLAN MAHADZIR ( $\sim$ dr.mdamahadzir@gmail.com )

Monash University https://orcid.org/0000-0003-1253-1426

Kia Fatt Quek

Monash University Tan Sri Jeffrey Cheah School of Medicine and Health Science

Amutha Ramadas

Monash University Tan Sri Jeffrey Cheah School of Medicine and Health Science

Research article

Keywords: Metabolic Syndrome, Nutrition, Lifestyle, Health Belief Model, Focus Group Discussion

Posted Date: February 25th, 2020

DOI: https://doi.org/10.21203/rs.2.24541/v1

License: (c) (1) This work is licensed under a Creative Commons Attribution 4.0 International License.

Read Full License 


\section{Abstract}

\section{Background}

Nutrition and lifestyle behaviour of individuals have been associated with the risk of metabolic syndrome (MetS). In order to better control the prevalence of MetS nationwide, an effective health promotion program must provide age-appropriate care information that addresses critical factors such as physiological function, social psychology, and emotional and health literacy in order to empower these patients to self-manage their condition and to enhance their self-care-related motivation and confidence. This study qualitatively analysed FGD involving Malaysian adults with MetS to explore their understanding of MetS and their perceived motivation and barriers of healthy nutrition and lifestyle behaviour.

Methods

A qualitative study with four focus groups (21 purposively sampled respondents) was conducted with adults diagnosed with MetS whom attending MONASH Medical Precinct in 2018. An interview protocol consolidating both responsive interviewing model and health belief model were prepared. The focus groups were audio recorded and transcribed. The data were analysed by emergent themes analysis. Data saturation was achieved in the fourth focus group.

Results

Seven main themes; three motivations and three perceived barriers and one subtheme on healthy nutrition and lifestyle behaviour were identified in the analysis of FGD. Motivations for healthy nutrition and lifestyles behaviour were (i) weight gain and physical appearances; (ii)personal experience of adverse complications and (iii) good family and social support. The identified perceived barriers were (i) healthcare as a business model; (ii) healthy change is difficult and expensive and (iii) cultural influence on food intake. Inadequate knowledge on MetS as most respondents were unaware of MetS as a clustering of risk factors but were able to identify the components individually was also a strong theme identified in this study.

Conclusion

Nutrition and lifestyle behaviours of adults with MetS were highly affected by the several potent motivations and perceived barriers among individuals. Information delivery and lifestyle promotion should address these aspects to increase program adoption and adherence, ensuring success of a community-based lifestyle intervention.

\section{Background}

Unhealthy dietary behaviour and lifestyle factors such as sedentariness, alcohol and tobacco use, and sleep deprivation, may lead to poor health. Nutrition and lifestyle behaviour of individuals have been 
associated with the risk of metabolic syndrome (MetS) (1). MetS is a clustering of cardiovascular risk factors which places individuals at increased risk for cardiovascular morbidity and mortality $(2,3)$. The increase in MetS prevalence among Malaysian adults for the past decade (4-6) reinforces the need of an effective health promotion program with age-appropriate care information that addresses critical factors such as physiological function (6), social psychology (7), and emotional and health literacy (1). Such programs can empower these patients to self-manage their condition and to enhance their self-carerelated motivation and confidence.

An exploration of models for individual motivation to participate in intervention measures is necessary to develop hypotheses regarding the influence of various factors on participation (8). For instance, theoretical frameworks such as Health Belief Model (HBM) was used to explain individual's behaviours and behavioural changes $(9,10)$. Furthermore, a deeper understanding on individuals' motivation and barriers to behaviour is among the key to ensure the success of lifestyle intervention measures (1). The HBM is made up of four constructs representing the perceived threat and net benefits: perceived susceptibility, perceived severity, perceived benefits, and perceived barriers, which accounted for individual's "readiness to act." (11). Besides to better fit the challenges of changing habitual unhealthy behaviours, such as being sedentary, smoking, or overeating, cues to action and self-efficacy concept are added into the construct. Cues to action would activate that readiness and stimulate overt behaviour while self-efficacy is to instil one's confidence in the ability to successfully perform an action (Fig. 1) (12, 13). Overall, HBM is the best model to explain the motivation and barriers faced by individuals in order to initiate a healthy behavioural change (10).

Consequently, we have to better characterised and understand the lifestyle behaviour of Malaysian adults with MetS before developing an effective community-specific lifestyle intervention. Since our understanding on behavioural determinants that shaped the lifestyle behaviour among Malaysian with MetS is limited, this study aimed to explore the abstract information on nutrition and lifestyle behaviours through qualitative analysis of focus group. This study will qualitatively analyse a series of focus group discussions (FGD) involving Malaysian adults with MetS to explore their understanding of MetS and their perceived motivation and barriers of healthy nutrition and lifestyle behaviour. Furthermore, HBM will be used as the conceptual model in the design of focus group question and overall script as it is the best model to chart out the awareness level and readiness of change among individuals (10).

\section{Methods}

Respondent

This study received ethical approval from Monash University Human Research Ethics Council (MUHREC) prior to respondent recruitment; (Project ID: CF16/56-2016000022). Individuals attending MONASH Medical Precinct were invited for MetS screening. Respondents were recruited using purposive sampling that sought out based on the MetS status which is the focus of the study. All consented respondent 
whom fulfilled the Harmonised criteria (14) of MetS were invited to join the FGD led by a moderator. Sociodemographic details and MetS components were recorded for all respondents.

Focus Group Discussion

Each session was decided based on the number of consented respondents which have to make a minimum group size of three. Moderated interviews were then arranged with respective groups. Four separate FGD sessions involving a total of 21 respondents took place in a private consultation room at Monash Medical Precinct. Each session was led and recorded by a moderator accompanied by a trained assistant. Moderator will use an interview protocol written based on responsive interviewing model (15). A protocol which consisted of a list of questions was developed based on Health Belief Model (HBM) domain framework (16) was used to introduce areas for open discussion. Since this study is keen to see how respondent would interact during the focus group, group dynamic and interactions were enhanced by reassuring all respondents that any views expressed in the focus group would remain confidential and that there were no 'right' or 'wrong' answers to the questions and that constructive criticism was a valued part of the process. Overall, the group structure would be a more compelling approach in evaluating one's lifestyle behaviours. As explained in an earlier study, the group dynamics response, that is the type and range of data generated through the social interaction of the group, can be deeper and richer than those obtained from one-on-one interviews (15).

Thematic Framework Analysis

All FGD session recordings were transcribed verbatim before undergoing thematic analysis. The steps of the thematic framework analysis $(15,17)$ were implemented using a priori issues derived from HBM constructs. Two initiation HBM components, motivation and perceived barriers towards healthy lifestyle, were used as primary domains in the thematic framework to facilitate textual, structural and composite descriptions of lifestyle behaviours among Malaysian adults with MetS; what do they know, what do they choose to do. Detection of salient phrases from the transcript followed by inductive coding was conducted line by line in each transcript. Comparison of codes was done both within and between the four transcripts with a constant reference was made to the coding framework and the study aim. Themes were developed by comparing codes within a category and by constant comparison of the codes across categories. This was done to ensure the interpretations remain grounded in the themes and its codes. In addition, to make sense of individual codes, relationship between the codes and the links between them were sought as a whole, to provide explanations for the findings and overarching themes. Hence, after an analysis of the transcripts, themes were categorized into the a priori domain as informed by the HBM concept.

\section{Results}

Sociodemographic Characteristic of Respondents 
Respondents $(n=21)$ who fulfilled the Harmonised MetS criteria participated in the study. On average participants were $51(S D=10.3)$ years old, ranging in age from 26 to 64 . The majority were women $(73 \%)$, married (73\%) and all had equal to or more than a high school education. Slightly less than half $46 \%$ were employed full-time. All participants had abdominal obesity according to the Harmonised Criteria $(14,18)$.

Table 1: Sociodemographic characteristic of study respondents. 


\begin{tabular}{|c|c|c|c|}
\hline \multicolumn{4}{|c|}{ Sociodemographic Characteristic } \\
\hline No & Item & $\mathrm{n}$ & $\%$ \\
\hline 1 & Age (years) & 21 & $51 \pm 10.3$ \\
\hline 2 & Gender & & \\
\hline & Male & 6 & 27 \\
\hline & Female & 15 & 73 \\
\hline 3 & Status & & \\
\hline & Single & 1 & 4 \\
\hline & Married & 15 & 73 \\
\hline & Widow & 3 & 14 \\
\hline & Divorced & 2 & 9 \\
\hline 4 & Ethnicity & & \\
\hline & Malay & 20 & 95 \\
\hline & Chinese & 1 & 5 \\
\hline 5 & Education & & \\
\hline & Secondary & 5 & 24 \\
\hline & Tertiary & 16 & 76 \\
\hline 6 & Occupation & & \\
\hline & Employed & 9 & 43 \\
\hline & Unemployed & 1 & 5 \\
\hline & Business Owner & 6 & 29 \\
\hline & Retired & 5 & 23 \\
\hline 7 & Income (MYR) & & \\
\hline & 0-999 & 1 & 5 \\
\hline & 1000-1999 & 2 & 9 \\
\hline & $2000-3999$ & 5 & 24 \\
\hline & $4000-5999$ & 5 & 24 \\
\hline & $6000-9999$ & 4 & 19 \\
\hline & $>10,000$ & 4 & 19 \\
\hline
\end{tabular}




\begin{tabular}{|c|c|c|c|}
\hline & \multicolumn{3}{|c|}{ Sociodemographic Characteristic } \\
\hline \multirow[t]{6}{*}{8} & \multicolumn{3}{|c|}{ Metabolic Risk Factors } \\
\hline & Abdominal Obesity & 21 & 100 \\
\hline & Hypertension & 21 & 100 \\
\hline & Diabetes & 19 & 91 \\
\hline & Dyslipidemia & 7 & 33 \\
\hline & Hypertriglyceride & 9 & 43 \\
\hline 9 & \multicolumn{3}{|c|}{ Number of risk factors } \\
\hline & 3 risks & 3 & 14 \\
\hline & 4 risks & 15 & 72 \\
\hline & 5 risks & 3 & 14 \\
\hline
\end{tabular}

Themes

Six strong themes emerged during the analysis of motivation and perceived barriers among adults with MetS. While one theme on perceived threat emerged on further analysis.

Motivations

Theme 1: Weight Gain and Physical Appearances

Participants expressed the changes in lifestyle is highly motivated by the gradual weight gain as they aged. The weight gain decreases their physical appearances resulted in them investing in anti-ageing products and food supplements. However, six participants extended their health monitoring by improving dietary habit and increasing physical activity. One of them said;

"You can eat all the pills but you will see the difference only once you sweat and eat better."

Weight gain is seen as a health threat as well especially among male respondents. They raised their concern on the worsening knee pain, shortness of breath and constant fatigue. Two of the participants said;

"the tummy has become large until I feel tired carrying it."

"you know your body is having problem when you have shortness of breath after climbing the stairs and feel extremely sleepy past the afternoon lunch"

While weight gain is a concern, female respondents are more concern on the skin and physical beauty. Observable changes on their skin prompted the female participants to eat better and exercise. One of the 
respondent said;

"I only started exercising when my dermatologist said it helps to get rid of my wrinkles."

Theme 2: Personal Experience of Adverse Complication

When being asked about what their view on MetS which accounts to increased risk of cardiovascular diseases and type 2 diabetes mellitus, participants shared their fears of being less healthy, or of being in such poor health that they could not continue in their normal roles, and how disability may affect their life. Four participants told stories of close family and relatives whom they knew who had strokes and rendered paralysed prior to it and how these had limit them. The participants expressed that they did not want their health compromised to the extent that they could not take care of themselves or their families. Hence, they are motivated to change to better. As one of the women said, when talking about all the things she still planned to do in life.

"I've made up in my mind but I'm not ready to go yet as I am not sure how to start."

The following quote is from a participant who shared how providing care for dad who got paralysed prior to stroke attack. Hence it has motivated her to make changes:

"Couple years ago, my father got stroke at 59 years old. Couldn't move, couldn't eat, couldn't do nothing and depend on us (the children) ...it really started all of us thinking and decided to change. Whatever that goes inside my husband and my children mouth is taken care of. It will be too late when we got stroke. Why want to trouble everyone around us because of our bad habits..."

Although several participants acknowledged how fear motivated them to action, two of the participants expressed opposite views indicating that fear alone was not enough to sustain lifestyle changes. One person commented that threat of poor health concerned her, but she didn't feel threatened enough to change the way she lived.

"If something destined to happen, it will happen, no matter how you take care."

Theme 3: Good Family and Social Support

The participants were asked about the environment and surrounding that may affects their life choices and habit. Eight of them voiced the need for support when making lifestyle changes. Different individuals expressed different type of support that they need or had received. Three participant talked about family members walking with her daily while another shared walking her dog daily routine. Participants gave examples of how individuals and pets had given primarily positive support. One of participant however shared that her husband prevents her to do the walking because he feels intimidated by her healthy lifestyle. Henceforth, the need to have friends or family support the person in making lifestyle changes was a strong theme. Five comments from participants were: 
"I don't have that support, I get discouraged and then, you know, I stop. If I had somebody to walk with me, I believe I could walk a little bit."

"I think that, means a lot when you got somebody else that's in the house that's take care of food and drinks and conscious about food every day."

"I always join my neighbour to walk around the neighbourhood. Usually we'll do it in a group. I feel very moving to go for a walk. My wife and kids are together too. On weekends, we usually have barbeque, get together. It's that kind of support that makes me feel better about myself."

If one does not have support it can be especially challenging as evident in this quote from a participant:

"Dieting is hard for a housewife like me. I have to take care of what my husband and my kids want to eat. My husband never cares about his food. I have to cook everything that he likes which is sometimes not a healthy choice. When it comes to exercise, we do it seldom. When my husband feels lazy, my kids will follow him. I usually walk with my neighbour, seldom, once a week."

"My husband is a jealous type. If he don't likes it, you better not do it. Like walking and all, he can never see me doing it. Because when I do it and he's lazy, he feels intimidated."

Barriers

Theme 4: Healthcare as a business model

There is an intricate relationship between participants and their health care providers. Most of the participants has been visiting Medical Precinct for more than two years. Trusting health care providers was important for them and most of them were told stories of doctors prescribing medications without discussing side effects, why the medications were needed. Five of the participants told that they are more interested on non- pharmacological options such as diet and exercise to reduce the risk of their diseases which however was not being provided by their previous health care providers. Most participants described relatively little or no discussion with health care providers about healthy eating and exercise, and they perceived doctors were rushed during visits.

"I always heard 'you are what you eat', but doctors are busy prescribing medications only."

When asked about nurse or diet counselling, none participants shared experiences either positive or negative impact of the counselling from within the private or public practice offices. They are more interested on the information provided by their medical doctors than other health personnel. Public has to be educated on what roles of each health personnel are playing. Dependency on medical doctor only will result to a long queue in health practices and delayed treatment.

"I don't think these people (nurse and dietician) understands what is happening inside me. They only speak based on their working manual. That's why I think it is crucial for doctor to explain more to us." 
Moreover, several participants voiced concerns that some illnesses were being used to financially benefit companies. This led to distrust for most participants, and communication and trust with their health care providers was a concern. One participant commented;

"...sometimes when the doctors prescribed too many medications, they act like they work for the pharmacy."

Two others commented:

"Doctors will tell all Indian people over 40 or 50 will high blood pressure. I think that is an easy statement for all (vague generalisation)."

"I think doctors give too much medication until five or six different medicines something's going to cancel out something."

Some of these statements portrayed a lack of understanding among participants on the diseases and its clinical management. For an instance, MetS itself has five different cardiometabolic risk factors that may require different medications on each case. Henceforth, health care providers should work to build trust and clearly explain their reasons for treatment recommendations as well as the role played by nurse and dietician. All participants viewed good communication and personal contact as building blocks to establish trust in the patient-provider relationship. One gentleman shared about the relationship he had with his former physician;

"...I had a good Chinese doctor friend in a clinic in Sunway..... But he passed away last year. All of my family consult with him before. That kind of doctor is hard to find. He will sit with you and tell you one by one why you need to take your medications. He will tell you to go jogging every morning. He never rushes like a normal doctor especially those in the public government practice."

Theme 5: Healthy change is hard and expensive

Besides, participants were aware about the details of good dietary habit including food pyramid, food timing and calorie counts but they found it hard to apply it in real life due to the cost of healthier food options, lack of support to practice healthy diet and daily exercise and finally the availability of food may influence participant food choices. Participant however ignored the habit of reading nutrition panel in all processed food that they consumed. $70 \%$ of participants reported previous participation in lifestyle intervention focussing on weight loss. Despite that, they suffered decay in healthy habits after six months of the end of intervention due to lack of support from the close surroundings. Besides, the healthy habits are seen as hard and unrealistic to be applied in Malaysia due to availability and affordability of healthier food options, the tedious process of calorie counting, lack of surrounding support on healthy lifestyle behaviour and inadequate knowledge on healthy lifestyle.

One of common comment when participants were asked to rate their healthy life; 
"This one is hard to talk about, but I found it's hard to keep being healthy every day."

Many participants had made lifestyle changes for a while, but then went back to previous habits. Four participants have previous subscribed for an expensive weight loss program and failed to maintain their weight after 7 months. While, the others commented that the changes introduce into their life is too hard and drastic. They do not know how to manipulate the change to keep it more exciting.

"It's boring to weight food every day. It feels like a fishmonger in a market."

"I bought all the weight loss vitamins (supplements) for nearly 10,000 (MYR), it feels slimmer for a while but after a year I feel my body expands."

For many, it was hard to continue to make life style changes related to healthy eating and increased exercise. The participants really viewed changing eating and physical activity for the long term as a "battle" and something they had to control. One participant pointed out that

"Once you have been off a diet and go back, it's just harder to go back."

"Once you started dieting and you fry a piece of chicken, you're going to fry some chicken every day for a week, and your diet just gone."

This process of beginning to make changes "getting on track" was viewed as very difficult. Likewise, if participants returned to old habits, it was hard to get "back on track," eating healthy foods and increasing physical activity. The feelings of discouragement of relapse came through very clearly in all sessions. One participant described getting off track as:

"My problem is common for everyone, I'm going to get up for exercise every morning, which I did initially and after that God knows. And then when I try to schedule it for afternoons and do it for a while, but I always feel tired so I don't go jogging that day."

Another participant shared her way of making sure that she gets her exercise:

"I'm always home until lunch, if I'm watching TV then I will cycle, If I can watch TV, I can exercise."

Participants were asked what it takes to be healthy and most of them answered that they do not know. They see as being healthy is to eat proper food which is more expensive than the easy local food and to be involved in active lifestyle which has the most people gave up. However, two participants give new perspectives on healthy lifestyle;

"Buy a weighing scale and a long mirror. Put it at your bedside. Every day, if you are fat, you can see you are fat."

"Health lifestyle needs discipline. That's why a lot of successfully people has a healthy life. They have the discipline to be healthy." 
Theme 6: Cultural influence on food intake

All respondent assume healthy diet is a plate filled with vegetable, organic food and drizzled with olive oils. Most respondent recognised 'Mediterranean Diet' as the perfect healthy diet. Thus, most of them feels like they are 'cheating' when they eat local foods. Several respondent gave up eating healthily at all because they were told to avoid rice;

"We as Malaysian can never stop eating rice. Make rice healthy and then only tell us to diet."

A Chinese female respondent claimed her diet to be different than other race. She said;

"We are told what to eat since we are kids. If you are Chinese, you will eat more soup and not eating at night. It's different than Indian, Malay and any other race in Malaysia."

However, one respondent said that despite the cultural influence in food choice, the current availability of foods in Malaysia has made choice is the only thing to blame. He mentioned;

"Now you can find most of the food you want to eat, Chinese, Indian, Malay, Italian, French, Japanese, Korean and all. You just need to know which to choose."

Perceived Threat

Theme 7: Inadequate knowledge on Metabolic Syndrome

In terms of perceived threat and susceptibility, participants were able to identify the components of MetS individually and relate it to the bad lifestyle and dietary habit. For example, high carbohydrate diet towards progression of diabetes and high salt intake that is responsible to cause hypertension. In general, participants were unfamiliar with MetS as a diagnosis. Most were unaware of the term and had not been told by their doctors that their combination of health problems was referred to as "Metabolic Syndrome".

"we only know about diabetes, high blood pressure and high blood cholesterol. We were only told to eat properly, exercise and eat our medication. Metabolic syndrome is new. If you said it's the big tummy problem, that's what I call fat."

Based on their individual risk factors, participants were aware of the need to make lifestyle behaviour changes to improve their health. In addition, they had knowledge of appropriate behaviours in which they should be engaged. For example, several were aware that the appropriate healthy serving plate concept; Quarter, Quarter and Half (19). However, they expressed a need for skill building to successfully make behaviour changes.

\section{Discussion}

HBM is the earliest theory developed to explain the process of lifestyle adoption and individual's behavioural choice. In the theory, three domains are suggested as the initiator in lifestyle and behaviour 
choices; motivations, perceived barriers and perceived threat (16). This study adopted HBM as the fundamental framework to understand nutrition and lifestyle behaviour of Malaysian adults with MetS. Findings from focus group analysis suggested three themes in each motivation and perceived barrier domains. Interestingly, in further analysis of themes, inadequate knowledge of MetS was found to be repeated which explains the perceived threat that underlies HBM concept and it is made as the seventh theme found in this study.

Weight gain and appearance is found to be one of the best motivation to adopt healthy lifestyle in our study. Gradual weight increase and localised fat distribution around central region among middle-aged respondents are hypothesised due to aging (20). This gradual weight gain increase the risk of fracture and disability $(21,22)$. Furthermore, weight gain also has been found to reduce quality of life resulting in increased concern of adopting healthy lifestyle $(20,21)$. Abdominal obesity is also raising a concern among respondents. Despite poor physical appearances due to large tummy, abdominal obesity also increases the risk of multitude metabolic complications (23). Consequently, targeted interventions may exploit this concern by addressing the benefit of weight loss in term of physical appearances and healthy ageing. This is also suggested that intervention that target physical changes such as weight loss are more favourable as compared to blood parameters thus will increase interest and hopefully adherences among adults with concerning lifestyle-related condition such as MetS.

Besides, our respondents whom experienced adverse health complications are also highly motivated to adopt healthier lifestyle. A few qualitative studies done on stroke patients $(24,25)$ and dialysis patient (26) also found relationship between experiences on health complications tend to make patients to more vigilant about their life choices. Moreover, respondent with family members whom affected by complications were also tend to become healthier. Earlier study has also found the same theme and conclude that they take by the lesson of observing poor quality of life affecting their family members (27, 28). During the analysis of this theme, an overarching theme were sought; limited knowledge on MetS among respondents. Most respondents were unaware of MetS as a clustering of risk factors but were able to identify the components individually. As a result, lifestyle changes come later in life which is supposedly may act as an effective prevention step from MetS $(2,29,30)$.

Another motivation of healthy lifestyle is to have good family and social support. Individuals residing in a neighbourhood with active community body tend to engage in weekly physical activity. Surroundings with public parks, safe pedestrian walkway and gymnasium likely to increase the physical activity levels of adults. Community engagement however is only limited on physical activity as nutrition and dietary behaviour is more likely to be influenced by family members (31). Thus supportive family members are found to be as the biggest motivator in adopting healthy or bad lifestyle behaviour (25). Besides, a few respondents reported supportive peers are among the reason why they adopt better diet and lifestyle daily especially among pensioner. Studies involving peer support has found that peer may influence they group to adopt better lifestyle due to the same exposure towards a certain health condition and experience the same surroundings that may encourage better activity engagement among them (32-34). 
In term of barriers, respondents assumed healthcare, especially preventative and primary care is a business model. This finding raised a red flag on general misunderstanding that aid the inaccessibility of primary care to reach the population. Patients argued that preventative medicine and primary care intentionally create the need to seek early but unnecessary medical attention. Hence, the perceived risk and threat are believed to be exploited in pharmaceuticals and nutraceuticals industry. Studies from India (35) and Singapore (36) elucidate an unnecessary fear of regular check-ups among healthy elderly because of conflicting fear of spending more on expensive healthcare. Thus, intervention needs to emphasise on the benefit of healthy lifestyle in term of disease prevention as well as improvement in individual's quality of life.

There is a perception that healthy lifestyle is tedious and expensive. Respondents were concern that tedious step in healthy meal preparation, healthy food choices and gradual increase in physical activity is hard and unsustainable. A study found out that a major barrier in integrating a lifelong healthy habit is the limitation to understand and translate available health information into easy and practical steps in daily life (37). Besides, another study found out that easy and realistic health goal setting plays a crucial role in sustainable lifestyle changes (38). Similar to this study, a respondent mentioned that to translate Malaysian Food Pyramid (19) onto a plate is confusing and hard. Furthermore, respondent understood healthy food as green choices, organic products and non-GMO products instead of food groups and food variety. Some studies explained that this has impacted on their food choices as they assumed healthy food as more expensive than their normal diet $(38,39)$.

As Malaysia is a multiracial country, different race in Malaysia has different preferences in their food choices and lifestyle behaviour. Population based studies has found that Malays, the majority race in Malaysia, tend to have high fat and high carbohydrate diet daily $(5,40)$. These studies found that cultural background tend to influence food choices of individuals. This racial discretion were found in other study as well; Chinese population has been found to be more perceptive towards healthy lifestyle as compared to Malays and Indians $(5,6)$. Accessibility to healthcare and predisposing literacy were argued as most important factors that influence better health among Chinese in Malaysia (6). However, given that MetS is very prevalent among Malay and Indian in Malaysia $(5,6,40)$, similar study involving Indian is warranted to obtain a better picture on their unique behavioural determinants.

Upon further analysis, inadequate knowledge on MetS is often repeated by the respondents, suggesting it as a strong theme to be addressed. This is in line with HBM as it is suggested that three main initiators of healthy lifestyle choices are motivation to change, perceived barriers of changes and perceived threat on diseases (13). Respondent were uninformed about MetS as a threat, thus they view these components individually instead of being concerned with its 'clustering'. Even though the management of MetS will target each component individually, informed perceived threat on clustering risk factors may improve perception of change among individuals as found in a study in Japan (41).

Collectively, targeting MetS, a lifestyle-related disease, warranted a holistic point of view. The intervention has to be specifically designed to the need of change by delivering a basic understanding of diseases, its 
severity and individual's susceptibility of acquiring it. Besides, the adoption of healthy lifestyle is usually limited by behavioural determinants such as motivation and perceived barriers. In this study, we found that motivation such as gradual weight gain, physical appearances, adverse health complication experiences and good social support will increase the adoption of healthy lifestyle. While several perceived barriers such as perception of healthcare as business model, difficult and expensive lifestyle changes and cultural influences on food choices hindered the adoption of better nutrition and lifestyle behaviour. Finally, inadequate of knowledge on MetS abolished the need to change among individuals. Since the prevalence of MetS among Malaysian adults has increased tremendously for the past decades, an effective community-based lifestyle intervention is demanded to manage and further prevent MetS to affect the country.

Study Limitation

The qualitative approach, the selectness of the population under study and the sample size are clear limitations of this study. As opposed to studies taking a phenomenological or grounded theory approach, qualitative descriptive studies that involves focus group may be less interpretive, however, they are superior than quantitative studies (15). Despite, in the development of community-based lifestyle intervention, there is no one-size-fits-all solution. Thus addressing the qualitative components of a community that are often missed out in quantitative observation, may increase the acceptability of any lifestyle intervention among the community members (17). As the consent was sought and the confidentiality of all respondent is protected, the study poses no serious ethical question.

\section{Conclusion}

The qualitative insight informed by a thematic qualitative analysis of FGDs In this study has generated an perception of change in Malaysian adults towards healthy lifestyle. Individual perceptions on disease threat, physical appearances, adverse health effect, functionality of healthcare system and benefit of changes will significantly modulate the need to change in them. Besides, supportive environment will increase the degree of adoption and sustainability of healthy lifestyle among individuals. In conclusion, the results support the practicability and value of the study in informing a better lifestyle intervention development targeting Malaysian adults with MetS.

\section{Abbreviation}

MetS Metabolic syndrome

FGD $\quad$ Focus group discussion

SD Standard deviation

MYR Malaysian Ringgit 


\section{Declarations}

\section{Ethics and Consent to Participate}

Ethics approval was obtained from Monash University Human Research Ethics Council (MUHREC) prior to respondent recruitment; (Project ID: CF16/56 - 2016000022). Participants consented their participation by signing a Study Consent Form.

\section{Consent to Publish}

Not applicable

\section{Authors Contribution}

MDAM designed the study and carried out the interviews and analysis. MDAM drafted the manuscript in cooperation with AR. MDAM and AR participated in the design of the study and helped to draft the manuscript. All authors reviewed and agreed on the final manuscript. The study adheres to the RATS guidelines on qualitative research. All authors read and approved the final manuscript. Muhammad Daniel Azlan Mahadzir, Quek Kia Fatt and Amutha Ramadas contributed equally to this work.

\section{Funding}

The research received financial support from Fundamental Research Grant Scheme (FRGS) (Grant No: FRGS/2/2013/SKK07/MUSM/03/1) from Ministry of Higher Education Malaysia.

\section{Data Availability}

The datasets generated and/or analysed during the current study are not publicly available due identification tags on FGD excerpts but are available from the corresponding author on reasonable request.

\section{Competing interests}

The authors declare that they have no competing interests.

\section{References}

1. Martin CA, Gowda U, Smith BJ, Renzaho AMN. Systematic Review of the Effect of Lifestyle Interventions on the Components of the Metabolic Syndrome in South Asian Migrants. J Immigr 
Minor Health. 2018;20(1):231-44.

2. Nyenwe EA, Dagogo-Jack S. Metabolic syndrome, prediabetes and the science of primary prevention. Minerva Endocrinol. 2011;36(2):129-45.

3. Londhe SS. A major health hazard: Metabolic syndrome. Int J Pharmcy Pharm Sci. 2011;3(3):1-8.

4. Ghee LK, Kooi CW. A review of metabolic syndrome research in Malaysia. Med J Malays. 2016;71:208.

5. Rampal S, Mahadeva S, Guallar E, Bulgiba A, Mohamed R, Rahmat R, et al. Ethnic Differences in the Prevalence of Metabolic Syndrome: Results from a Multi-Ethnic Population-Based Survey in Malaysia. PLoS ONE. 2012;7(9).

6. Tan AKG, Dunn RA, Yen ST. Ethnic disparities in metabolic syndrome in Malaysia: An analysis by risk factors. Metab Syndr Relat Disord. 2011;9(6):441-51.

7. De Sousa SMCD, Norman RJP. Metabolic syndrome, diet and exercise. Best Pract Res Clin Obstet Gynaecol. 2016;37:140-51.

8. Marks DF. The quest for meaningful theory in health psychology. J Health Psychol. 2008;13(8):97781.

9. Coleman MT, Pasternak RH. Effective Strategies for Behavior Change. Prim Care Clin Off Pract. 2012;39(2):281-305.

10. Bully P, Sánchez T, Zabaleta-del-Olmo E, Pombo H, Grandes G. Evidence from interventions based on theoretical models for lifestyle modification (physical activity, diet, alcohol and tobacco use) in primary care settings: A systematic review. Prev Med. 2015;76(S):S76-S93.

11. Finfgeld DL, Wongvatunyu S, Conn VS, Grando VT, Russell CL. Health belief model and reversal theory: A comparative analysis. J Adv Nurs. 2003;43(3):288-97.

12. Roden J. Revisiting the Health Belief Model: Nurses applying it to young families and their health promotion needs. Nurs Health Sci. 2004;6(1):1-10.

13. Clarke VA, Lovegrove H, Williams A, Machperson M. Unrealistic optimism and the health belief model. J Behav Med. 2000;23(4):367-76.

14. Ramli AS, Daher AM, Noor Khan Nor-Ashikin M, Mat-Nasir N, Keat Ng K, Miskan M, et al. JIS definition identified more malaysian adults with metabolic syndrome compared to the NCEP-ATP III and IDF criteria. BioMed Res Int. 2013;2013.

15. Wong LP. Focus group discussion: A tool for health and medical research. Singapore Med J. 2008;49(3):256-61.

16. Roden J. Validating the revised Health Belief Model for young families: Implications for nurses' health promotion practice. Nurs Health Sci. 2004;6(4):247-59.

17. Onwuegbuzie AJ, Dickinson WB, Leech NL, Zoran AG. A Qualitative Framework for Collecting and Analyzing Data in Focus Group Research. International Journal of Qualitative Methods. 2009;8(3):121. 
18. Zainuddin LRM, Isa NF, Wan Muda WM, Mohamed HJ. The prevalence of metabolic syndrome according to various definitions and hypertriglyceridemic-waist in Malaysian adults. Intl J Prev Med. 2011;2(4):229-37.

19. Health Mo. Malaysian Dietary Guidelines. In: Nutrition NCCoFa, editor. Kuala Lumpur2010.

20. Bredland EL, Söderström S, Vik K. Challenges and motivators to physical activity faced by retired men when ageing: A qualitative study. BMC Public Health. 2018;18(1).

21. Chen WC, Lin CC. Constructing a peer-led self-management program for elderly patients with metabolic syndrome. J Nurs. 2017;64(5):111-9.

22. Gardiner S, Glogowska M, Stoddart C, Pendlebury S, Lasserson D, Jackson D. Older people's experiences of falling and perceived risk of falls in the community: A narrative synthesis of qualitative research. International journal of older people nursing. 2017;12(4).

23. Nikolopoulou A, Kadoglou NPE. Obesity and metabolic syndrome as related to cardiovascular disease. Exp Rev Cardiovasc Ther. 2012;10(7):933-9.

24. Wray F, Clarke D. Longer-term needs of stroke survivors with communication difficulties living in the community: a systematic review and thematic synthesis of qualitative studies. BMJ Open. 2017;7(10):e017944.

25. Pindus DM, Mullis R, Lim L, Wellwood I, Rundell AV, Abd Aziz NA, et al. Stroke survivors' and informal caregivers' experiences of primary care and community healthcare services - A systematic review and meta-ethnography. PLoS ONE. 2018;13(2):e0192533.

26. Reid C, Seymour J, Jones C. A Thematic Synthesis of the Experiences of Adults Living with Hemodialysis. Clinical journal of the American Society of Nephrology : CJASN. 2016;11(7):1206-18.

27. Sulaiman ND, Furler JS, Hadj EJ, Corbett HM, Young DYL. Stress, culture and 'home': Social context in Turkish and Arabic-speaking Australians' views of diabetes prevention. Health Promot $\mathrm{J}$ Aust. 2007;18(1):63-8.

28. Daivadanam M, Wahlström R, Ravindran TS, Thankappan K, Ramanathan M. Conceptual model for dietary behaviour change at household level: A 'best-fit' qualitative study using primary data. BMC Public Health. 2014;14(1).

29. Whyte J. The metabolic syndrome: Early clues, effective management. Consultant. 2005;45(11):1195-200.

30. Cooper-DeHoff RM, Pepine CJ. Metabolic syndrome and cardiovascular disease: Challenges and opportunities. Clin Cardiol. 2007;30(12):593-7.

31. Noor NM, Gandhi AD, Ishak I, Wok S. Development of Indicators for Family Well-Being in Malaysia. Soc Indic Res. 2014;115(1):279-318.

32. Embuldeniya G, Veinot P, Bell E, Bell M, Nyhof-Young J, Sale JE, et al. The experience and impact of chronic disease peer support interventions: a qualitative synthesis. Patient Educ Couns. 2013;92(1):3-12. 
33. Parry M, Watt-Watson J. Peer support intervention trials for individuals with heart disease: a systematic review. European journal of cardiovascular nursing : journal of the Working Group on Cardiovascular Nursing of the European Society of Cardiology. 2010;9(1):57-67.

34. Fisher EB, Boothroyd RI, Elstad EA, Hays L, Henes A, Maslow GR, et al. Peer support of complex health behaviors in prevention and disease management with special reference to diabetes: systematic reviews. Clinical diabetes and endocrinology. 2017;3:4.

35. Bhan N, Madhira P, Muralidharan A, Kulkarni B, Murthy G, Basu S, et al. Health needs, access to healthcare, and perceptions of ageing in an urbanizing community in India: A qualitative study. BMC Geriatr. 2017;17(1).

36. Tan NC, Cheah SL, Teo EK. A qualitative study of health-seeking behavior of Hepatitis B carriers. Singapore Med J. 2005;46(1):6-10.

37. Solbrig L, Jones R, Kavanagh D, May J, Parkin T, Andrade J. People trying to lose weight dislike calorie counting apps and want motivational support to help them achieve their goals. Internet Interv. 2017;7:23-31.

38. Enwald H, Hirvonen N, Huotari ML, Korpelainen R, Pyky R, Savolainen M, et al. Everyday health information literacy among young men compared with adults with high risk for metabolic syndrome A cross-sectional population-based study. J Inf Sci. 2015;42(3):344-55.

39. Wang CP, Li Y, Wang S. Health Literacy Evaluation Index System for Patients With Metabolic Syndrome From Clinical Perspective. Chin Gen Pract. 2016;19(10):1182-7.

40. Narayanan P, Meng OL, Mahanim O. Do the prevalence and components of metabolic syndrome differ among different ethnic groups? A cross-sectional study among obese Malaysian adolescents. Metab Syndr Relat Disord. 2011;9(5):389-95.

41. Kudo Y, Okada M, Tsunoda M, Satoh T, Aizawa Y. A lifestyle to prevent or combat the metabolic syndrome among Japanese workers: Analyses using the health belief model and the multidimensional health locus of control. Ind Health. 2011;49(3):365-73.

\section{Figures}

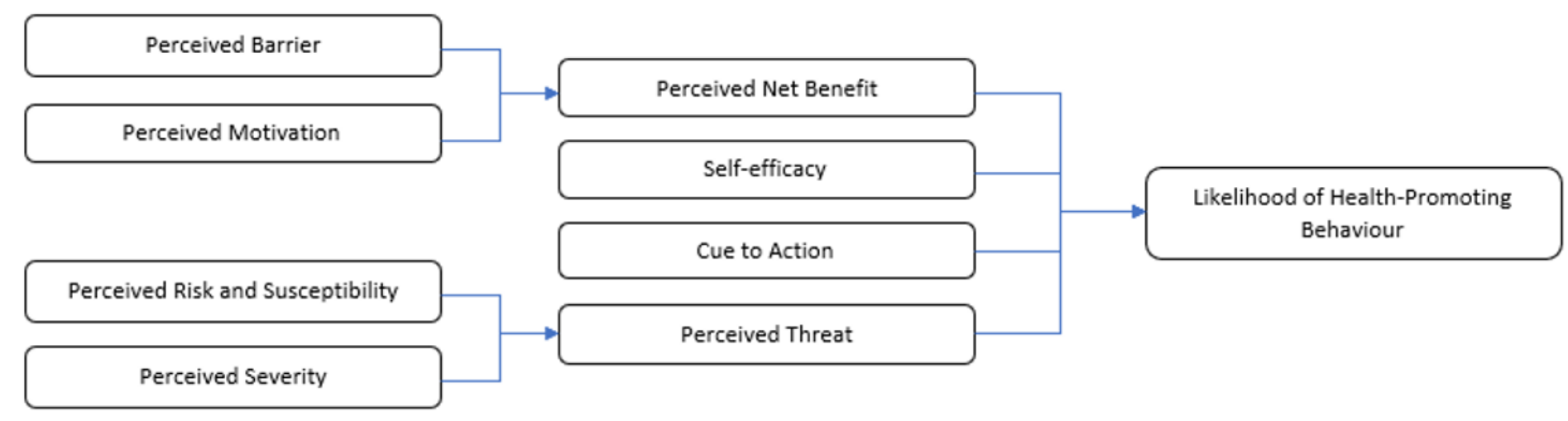


Figure 1

The Health Belief Model (HBM) (16) 